\title{
Prothymosin $\alpha$, a protein implicated in the proliferation and survival of lymphocytes
}

\author{
Manuel Freire ${ }^{1 *}$, Pablo Barbeito', Concepción S. Sarandeses', Cristina Díaz-Jullien', Juan Muras', Guillermo \\ Covelo', David Moreira' and Carmen Freire-Cobo' \\ 'The Department of Biochemistry and Molecular Biology, CIBUS, Faculty of Biology, University of Santiago de Compostela, 15782 Santiago de Compostela, \\ Spain
}

Article Info

\section{Article Notes}

Received: March 13, 2018

Accepted: April 13, 2018

\section{${ }^{*}$ Correspondence:}

Dr. Manuel Freire, Department of Biochemistry and Molecular Biology, CIBUS, Faculty of Biology, 15782

Santiago de Compostela, Spain,

E-mail: manuel.freire@outlook.es

(c) 2018 Freire M. This article is distributed under the terms of the Creative Commons Attribution 4.0 International License.

\section{Keywords:}

Cell proliferation

Cross-linking

Lymphocytes

Mitochondrial apoptosis

Protein complex

Prothymosin alpha

\section{ABSTRACT}

Prothymosin $\alpha$ (ProT $\alpha)$ is a $109-11$ amino acid protein widely distributed in mammalian tissues and particularly abundant in lymphoid cells. Genomic and proteomic studies led to consider ProT $\alpha$ as a multifunctional protein implicated in nuclear and cytoplasmic functions. The nuclear function of ProT $\alpha$ is related to chromatin activity through its interaction with core histones and proteins involved in chromatin remodelling, whereas, processes related to the phosphorylation, the proteolytic processing to generate Thymosin $\alpha 1$, and the role as anti-apoptotic factor of ProT $\alpha$, are linked to its cytoplasmic location. Affinity chromatography and co-immunoprecipitation experiments have demonstrated novel interactions of ProT $\alpha$ with acidic proteins such as SET, ANP32A, and ANP32B in the cytoplasm of proliferating lymphocytes. The stabilization of these interactions by chemical cross-linking with formaldehyde shows that they are formed through associations in six acidic complexes which correspond to selective interactions of SET and ANP32 proteins with ProT $\alpha$. These ProT $\alpha$-complexes also include cytoplasmic proteins implicated in membrane remodelling and in mitochondrial activity. In conclusion, these novel protein interactions of ProT $\alpha$ observed in proliferation activity and apoptosis studies, suggest that they might be related to mechanisms involved in the proliferation activity and the apoptotic control of lymphocytes.

\section{Introduction}

In 1966 White and Goldstein's group intended to identify protein factors which could be implicated in the biological function of the thymus and thus isolated a mixture of polypeptides named thymosin ${ }^{1}$. The best known thymosin preparation is a calf thymus extract named Thymosin Fraction 5 (TF5). The TF5 contains about 30 different heat-stable polypeptides showing immune regulatory properties in several assay systems ${ }^{2}$ in vitro and in vivo. The first purified, sequenced and most characterized component of TF5 is the Thymosin $\alpha 1$ (T $\alpha 1$ ), a polypeptide comprised of 28 amino acids. T $\alpha 1$ is 10-1000 times more active than TF5 in different immune assays ${ }^{3}$. Thymosin $\alpha 11$ (T $\alpha 11)$, another component of TF5 structurally related to $T \alpha 1$, was later isolated and sequenced ${ }^{4}$. The structure of $T \alpha 11$ corresponds to the sequence of $T \alpha 1$ plus seven additional amino acid residues at its $\mathrm{C}$-terminus. $\mathrm{T} \alpha 11$ is about four times less abundant than $\mathrm{T} \alpha 1$ in TF5, however, it shows similar activity to T $\alpha 1$ in some in vivo assays ${ }^{5}$. 
The strategy to characterize a precursor of the $\alpha$-thymosins in the thymic cells from translation products of calf thymus mRNAs led our group to isolate a protein which included $\mathrm{T} \alpha 1$ in its sequence ${ }^{6,7}$. This protein, isolated from rat thymus was later sequenced and named Prothymosin $\alpha(\operatorname{ProT} \alpha)^{8}$. The sequence of ProT $\alpha$ contains a $\mathrm{T} \alpha 1$ structure located at its first 28 amino acid residues and a T $\alpha 11$ structure located at its 35 amino acid residues. The primary structure of ProT $\alpha$ obtained from other mammalian tissues demonstrates that ProT $\alpha$ is an acidic protein with a highly conserved sequence containing between 109 and 111 amino acids. Figure 1a shows the sequence of human ProT $\alpha^{9}$, which includes a central

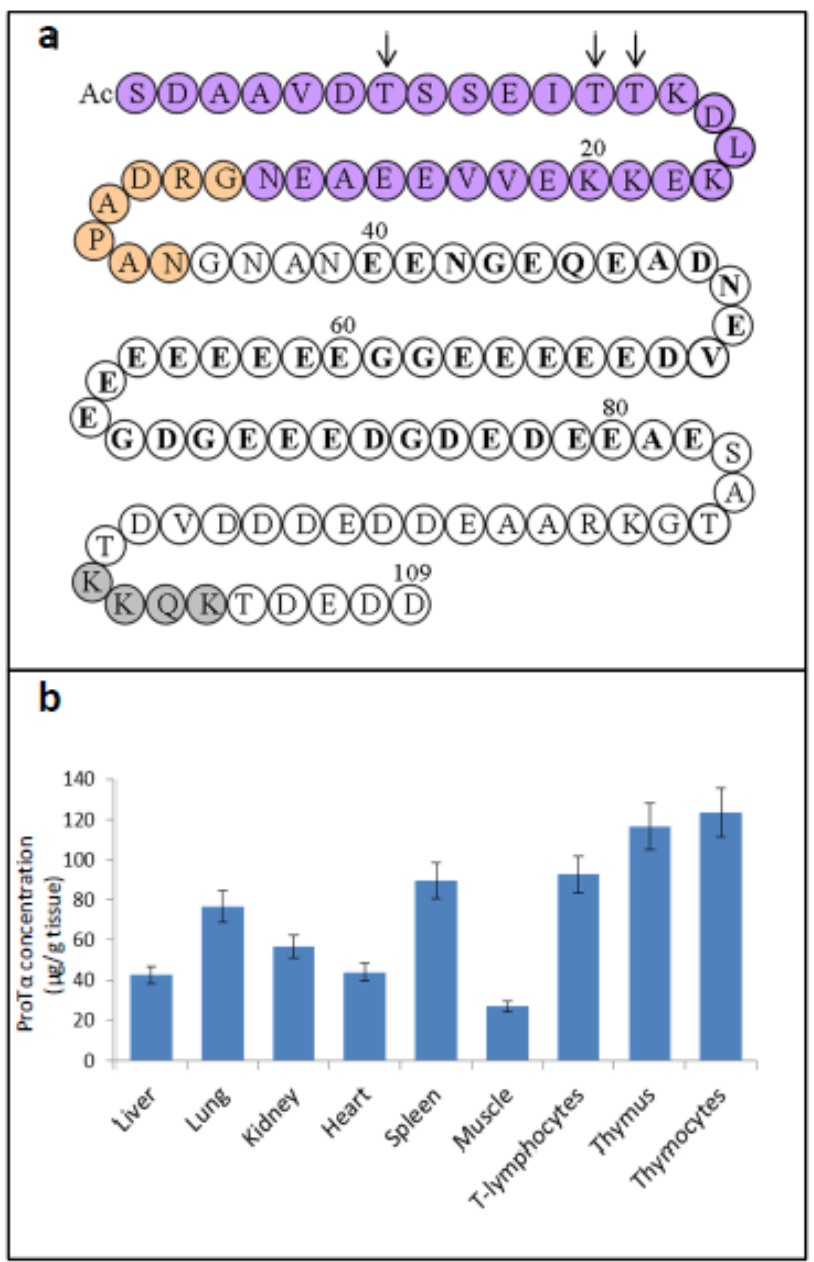

Figure 1. a) Human Prothymosin $\alpha$ amino acid sequence (1109) ${ }^{9}$. AcS indicates acetylated $\mathrm{N}$-terminal serine derived from the generalized co-translational processing which undergoes ProT $\alpha^{16}$. $T \alpha_{1}$ sequence is indicated in purple circles. $T \alpha_{11}$ sequence comprises the $T \alpha_{1}$ sequence plus an additional 7 residues at its $\mathrm{C}$-terminus (indicated in orange circles). Other structural characteristics of the ProTa sequence (referred in the text) are also indicated: arrows mark the phosphorylation sites of ProT $\alpha$ in normal (Thr7 and Thr12 or 13) and carcinogenic (only Thr7) lymphocytes; acidic region is indicated in bold type and nuclear localization signal is shown in grey circles. b) Concentrations of ProT $\alpha$ determined in extracts of different rat tissues ${ }^{11}$. acidic region (residues 40-82) with glutamic and aspartic residues and other structural characteristics.

Later studies on ProT $\alpha$ gene expression end the theory of an exclusive role of the $\alpha$-thymosins in the thymus immune function, as deduced from its generalized presence in different mammalian tissues ${ }^{10,11}$. The functional ProT $\alpha$ gene (PTMA) is located on chromosome 2 in humans ${ }^{12}$. A significative feature of ProT $\alpha$ is its high level of gene expression in proliferating cells ${ }^{13,14}$, on the range of core histones, especially in lymphoid tissues ${ }^{11}$. In Figure $1 \mathrm{~b}$ are summarized the concentrations of ProT $\alpha$ in different rat tissues.

\section{The biological function of ProT $\alpha$}

Further research on the intracellular evolution of ProT $\alpha$ provides important clues on its possible biological role. Characteristics of its gene structure and expression show the absence of a signal peptide ${ }^{15}$. Similarly, co-translational acetylation of its $\mathrm{N}$-terminal serine residue ${ }^{16}$ indicates that ProT $\alpha$ should have an intracellular site of action or at least that it can be secreted by a non-conventional mechanism. Studies on the subcellular distribution of ProT $\alpha$ demonstrate a preferential nuclear location in proliferating cells ${ }^{17,18}$ which is in agreement with the presence of a nuclear localization signal at the C-terminus of its sequence ${ }^{19}$. However, the prominent cytoplasmic presence of ProT $\alpha$ in cell lysates demonstrates a special ability of this protein to leak from the nucleus during the cell extraction process ${ }^{15,20}$.

The characterization of the components present in a nuclear-protein complex isolated by affinity to ProT $\alpha^{20,21}$ has served towards the elucidation of the putative mechanisms by which ProT $\alpha$ might be involved in the nuclear activity. This nuclear-protein complex is comprised of core histones, acetyltransferases of histone $\mathrm{H} 3$ and $\mathrm{H} 4$, the methyltransferase of histone $\mathrm{H} 3$, as well as transcription factors and other proteins implicated in chromatin remodelling ${ }^{22}$. Its presence in this complex supports a nuclear role of ProT $\alpha$ related with the chromatin activity.

In addition to nuclear activity, studies on the cytoplasmic evolution of ProT $\alpha$ also contributed to deepen the knowledge of its biological function. In this sense, data revealing the natural processing of ProT $\alpha$ to generate $\mathrm{T} \alpha 1$ and $\mathrm{T} \alpha 11$, and those showing the cytoplasmic phosphorylation of ProT $\alpha$ are especially relevant.

The lysosomal asparaginyl endopeptidase, mammalian legumain, is responsible for the processing of ProT $\alpha$ in vivo to generate $\mathrm{T} \alpha 1$ and $\mathrm{T} \alpha 11$, demonstrating the natural origin of these polypeptides ${ }^{23}$. This research also demonstrates that $\mathrm{T} \alpha 1$ is the main product in the cytoplasm, showing a concentration similar to that of ProT $\alpha$. Although potential immunotherapeutic effects of $T \alpha 1$ have been recently 
reported ${ }^{24}$, the mechanism of the biological role of this polypeptide remains elusive.

Cytoplasmic phosphorylation of ProT $\alpha$ in threonine residues is dependent on the proliferation activity ${ }^{25,26}$ and the carcinogenic transformation of the cells. Thus, residues Thr12 or Thr13 are phosphorylated in addition to Thr7 in normal lymphocytes while in carcinogenic lymphocytes Thr7 is the only residue phosphorylated ${ }^{27}$. The M2 isoenzyme of the pyruvate kinase is the enzyme responsible for the phosphorylation of ProT $\alpha$ that shows this novel dual function ${ }^{28}$.

A cytoplasmic function of ProT $\alpha$ as an anti-apoptotic factor has also been reported. ProT $\alpha$ seems to be related to the control of the apoptosome activity counteracting the pro-apoptotic action of the protein ANP32A (also known as PHAPI, pp32 or I1PP2A) to promote the caspase-3 activation ${ }^{29}$. Experiments using cells transformed with ProT $\alpha$ mutated to prevent its phosphorylation, indicate that phosphorylation of ProT $\alpha$ might be required for its anti-apoptotic activity ${ }^{18}$. This role of ProT $\alpha$ in the apoptotic control is shared by other acidic proteins like $\mathrm{SET}^{30}$ (also named TAF-I, PHAPII or I2PP2A) and ANP32B ${ }^{31}$ (also named PHAPI2, and APRIL). These proteins, and ANP32A, are nucleus-cytoplasmic shuttling proteins with a long stretch of acidic amino acids. They have a remarkable structural and functional homology with ProT $\alpha$ and a common ability to interact with histones and to influence the chromatin activity. In Figure 2 are shown their sequences.

Although implication of SET in the apoptotic control is well characterized, the mechanisms by which ProT $\alpha$ and the ANP32 proteins are implicated in this process remain elusive. Data from our laboratory, recently published in the Archives of Biochemistry and Biophysics ${ }^{35}$, reveal interactions of ProT $\alpha$ with SET, ANP32A, ANP32B and other cytoplasmic and mitochondrial proteins that shed some light on the mechanisms of a cytoplasmic action of ProT $\alpha$ and their targets. According to the actual data on the cellular site of action of ProT $\alpha$, reports showing extracellular effects of this protein should be questioned, at least until the mechanisms for its cellular secretion can be demonstrated.

\title{
SET
}

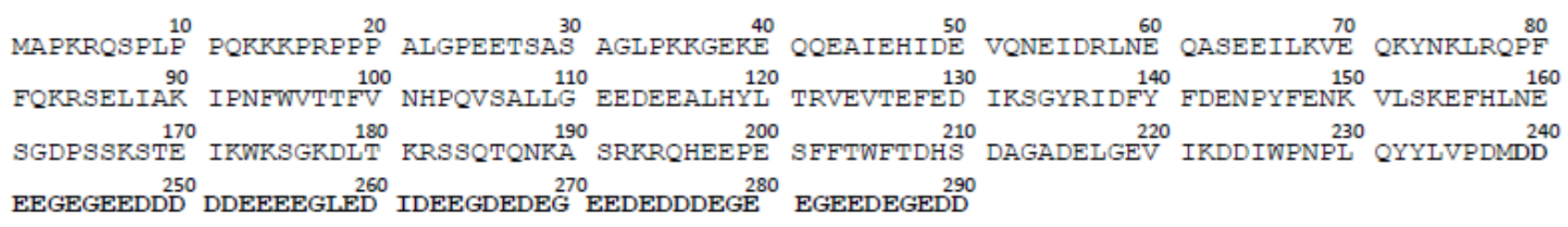

\section{ANP32A}

\begin{abstract}
MEMGRRIHLE LRNRTPSDVR ELVLDNSRSN EGRLEGLTDE FEELEFLSTI 10 NVGLTSIANL PRLNRLKRLE LSDNRVSGGI EVLAEKCPNL THLNLSGNKI KDLSTIEPLK RLENLRSLDL FNCEVTNLND YRENVFKLIP 1120 QLTYLDGYDR DDKEAPDSDA

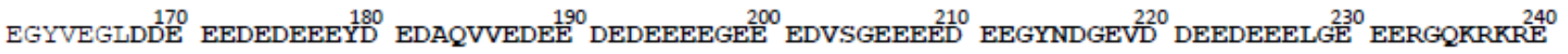
PEDEGEDDD 249
\end{abstract}

\section{ANP32B}

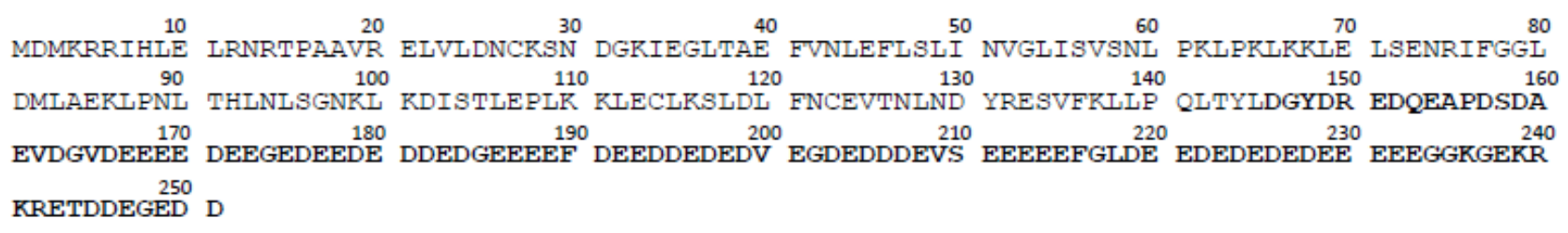

\section{ProTa}

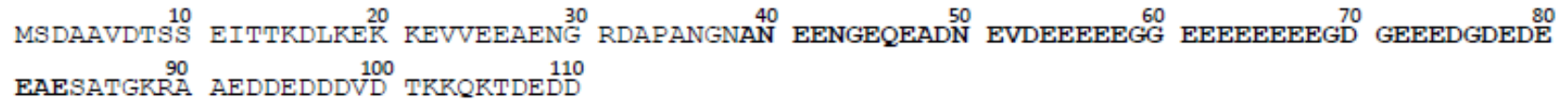

Figure 2. Comparison of the human sequences of SET, ANP32A, ANP32B and ProTa.

Stretches of acidic amino acid residues are indicated in bold type. Protein sequences were obtained from https://www.ncbi.nlm.nih.gov/ protein/. 
The interactions of ProT $\alpha$ with SET, ANP32A and ANP32B in lymphocytes

Affinity chromatography and co-immunoprecipitation experimentsperformed in subcellular fractions of transformed human T-lymphocytes (Jurkat cells) show a novel ability of ProT $\alpha$ to interact in vivo with ANP32A and ANP32B proteins in the cytoplasm of these cells. These results also confirm the ability of ProT $\alpha$ to interact with SET ${ }^{36}$.

Further experiments with Jurkat cells previously treated with formaldehyde to stabilize possible interactions by chemical cross-linking, lead to isolating six acidic protein complexes ranging from 25 to $45 \mathrm{kDa}$. These results confirm the ability of ProT $\alpha$ to interact with SET and ANP32 proteins.
The composition of the ProT $\alpha$-complexes (see Figure 3) denoted a different association of ProT $\alpha$ with SET (complexes $40 \mathrm{kDa}$ and $22 \mathrm{kDa}$ ), ANP32A and ANP32B (complexes 30, 28 and $25 \mathrm{kDa}$ ), and ANP32A (complex 34 $\mathrm{kDa}$ ). Other components in complexes of $40,34,30$ and 25 $\mathrm{kDa}$, are multifunctional proteins which would relate them with cytoplasmic functions such as: proteolytic processing, membrane trafficking, mitochondrial permeability and cell proliferation. The characterized interactions of ProT $\alpha$ with SET and ANP32 proteins reveal a significant capacity of these acidic proteins to associate. This property has been already stated in complexes in which interactions between SET and ANP32 proteins are involved in the apoptotic control $^{30}$ or in the regulation of histone acetylation ${ }^{37}$.

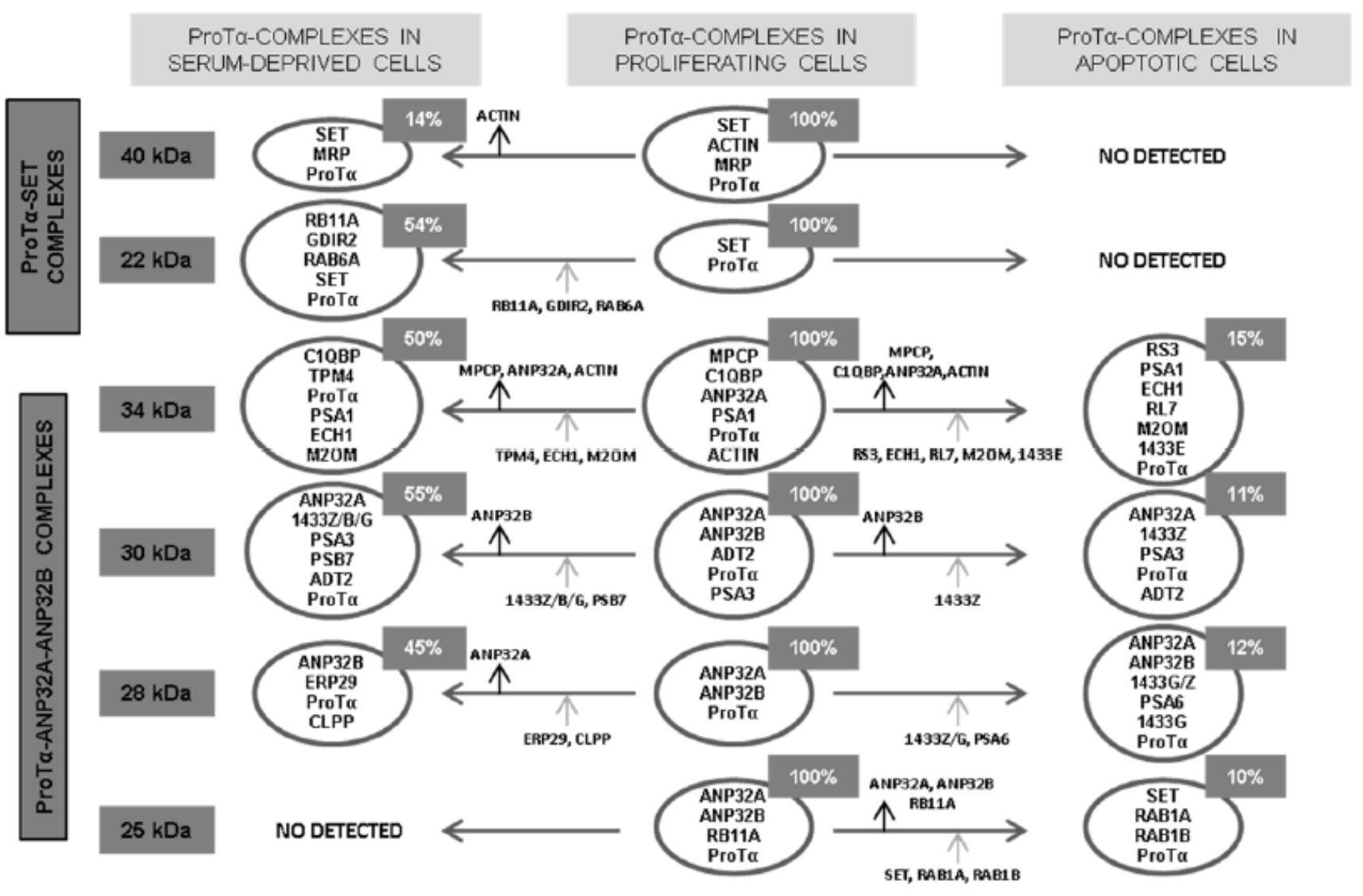

Figure 3. Scheme summarizes the composition of the ProT $\alpha$-immunoreactive complexes isolated from proliferating Jurkat cells, as well as their evolution when proliferating activity is reduced by serum deprivation or apoptosis induced by staurosporine.

Arrows indicate addition (grey arrows) or loss (black arrows) of proteins in the diverse complexes from proliferating cells when proliferation is reduced or apoptosis induced. Relative abundance of each complex is indicated in the upper insets. Acronyms of proteins other than ProT $\alpha$, SET, ANP32A, ANP32B and $\beta$-actin present in the different complexes: $40 \mathrm{kDa}$ : MRP (MARCKS-related protein); $22 \mathrm{kDa}$ : RB11A (Ras-related protein Rab-11A), GDIR2 (Rho GDP-dissociation inhibitor 2), RAB6A (Ras-related protein Rab-6A); 34 kDa: MPCP (Phosphate carrier protein, mitochondrial), C1QBP (Complement component 1Q subcomponent-binding protein), PSA1 (Proteasome subunit alpha type-1), TPM4 (Tropomyosin alpha-4 chain), ECH1 (Delta(3,5)-delta(2,4)dienoyl-CoA isomerase, mitochondrial), M2OM (Mitochondrial 2- oxoglutarate/malate carrier protein), RS3 (40S ribosomal protein S3), RL7 (60S ribosomal protein L7), 1433 E (14-3-3 protein epsilon); 30 kDa: ADT2 (ADP/ATP translocase 2), PSA3 (Proteasome subunit alpha type-3), PSB7 (Proteasome subunit beta type-7), 1433Z/B/G (14-3-3 protein zeta-delta/beta-alpha/gamma); 28 kDa: ERP29 (Endoplasmatic reticulum resident protein 29), CLPP (Putative ATP-dependent Clp protease proteolytic subunit), PSA6 (Proteasome subunit alpha type-6), 1433G/Z; 25 kDa: RB11A (Rasrelated protein Rab-11A), RAB1A/1B (Ras-related proteins Rab-1A and Rab-1B). 
Quantification in the affinity chromatography and co-immunoprecipitation experiments of ProT $\alpha$, SET and ANP32A and ANP32B proteins, as well as in the isolated complexes, indicate that only a small part of the cellular content of these proteins (about 10\%) is implicated in the characterized interactions. This is in agreement with the low cytoplasmic concentration of these proteins found in the immunofluorescence analysis of proliferating lymphocytes ${ }^{35}$. However, data from the evaluation of the subcellular concentrations of ProT $\alpha$, SET, ANP32 proteins in extracts from Jurkat cells show a surprising preponderant cytoplasmic presence of these proteins ${ }^{35}$. This effect might be due to their nuclear leaking produced during the homogenization procedure, as previously demonstrated with ProT $\alpha^{20}$.

The loss of interaction of ProT $\alpha$ with its targets is observed when cell proliferation of the Jurkat cells is reduced by serum deprivation or apoptosis induced by staurosporine. These effects seem to indicate that these interactions are related to cell proliferation and survival. In Figure 3 is shown the composition of the ProT $\alpha$-complexes and their evolution in serum-deprived and apoptotic cells.

The cytoplasmic functions attributed to the proteins interacting with ProT $\alpha$, like ANP32 $\mathrm{A}^{29}$ and $\beta$-actin ${ }^{38}$ leads to the hypothesis that ProT $\alpha$ might exert its anti-apoptotic effect counteracting the pro-apoptotic effect described for these proteins. On the contrary, and considering the anti- apoptotic activity of ANP32 ${ }^{31}$, the interactions with ProT $\alpha$ might reflect its role as a collaborative factor. In addition, interactions of ProT $\alpha$ with SET influencing cell survival might be related to different mechanisms of those attributed to SET as the anti-apoptotic factor through inhibition of the nuclease NM23- $\mathrm{H}^{30}$. On the other hand, interactions of ProT $\alpha$ with ANP32A/ANP32B in complexes 34 and $30 \mathrm{kDa}$, seem to be involved in mechanisms in which the mitochondrial activity is implicated.

\section{Concluding remarks}

Novel interactions described for ProT $\alpha$ provide clues for the understanding of the cytoplasmic activity of ProT $\alpha$ and its targets. The biological functions attributed to proteins interacting with ProT $\alpha$, lead to hypothesize that the ProT $\alpha$ complexes are implicated in cell survival by preventing the progress of apoptotic pathways in proliferating lymphocytes. This ability of ProT $\alpha$ supports its role as a multitasker protein with differentiated nuclear and cytoplasmic functions. The scheme in Figure 4 summarizes the cellular processes involving ProT $\alpha$ according to currently available data.

Most of the cellular ProT $\alpha$ migrates to the nucleus of proliferating cells to affect the chromatin activity. Cytoplasmic ProT $\alpha$ undergoes a proteolytic processing to generate $\mathrm{T} \alpha 1$ and a small part of the ProT $\alpha$ pool (about $10 \%$ ) is phosphorylated by M2-PK concomitantly with cell proliferation. A similar amount of ProT $\alpha$ is involved in the interactions with SET, ANP32A, ANP32B and other cytoplasmic and mitochondrial proteins. On this regard, it should be mentioned the possibility that phosphorylated ProT $\alpha$ could be implicated in such protein interactions as

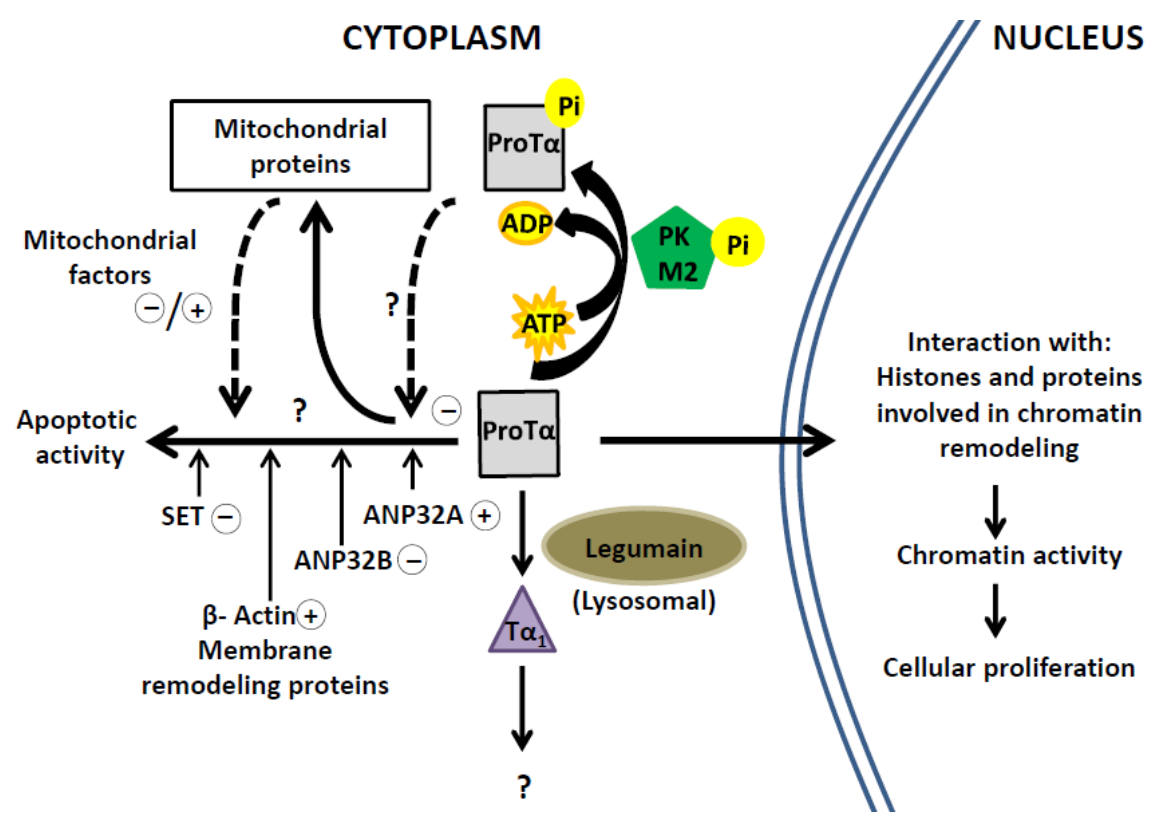

Figure 4. Pathways of Prothymosin $\alpha$ in proliferating cells related to nuclear and cytoplasmic activities through interactions with the indicated proteins.

Questions marks indicate insufficiently characterized mechanisms. Signs +/-, respectively, indicate assigned pro-apoptotic/antiapoptotic effects. 
deduced from the analysis of levels of effector caspases in cells transfected with ProT $\alpha^{18}$. However, we failed to detect ProT $\alpha$-phosphorylated peptides in the structural analysis of the ProT $\alpha$-complexes. This question should be addressed in future research to elucidate the functional mechanisms of ProT $\alpha$ and its targets.

\section{Disclosure}

The authors have declared that no conflict of interest exists.

This research did not receive any specific grant from funding agencies in the public, commercial, or not-forprofit sectors.

\section{References}

1. Goldstein AL, Slater FD, White A. Preparation, assay and partial purification of a thymic lymphocytopoietic factor (thymosin). Proc Nat Acad Sci USA. 1966; 56(3): 1010-1017.

2. Goldstein AL, Guha, A, Zatz M, et al. Purification and biological activity of thymosin, a hormone of the thymus gland. Proc Nat Acad Sci USA. 1972; 69(7): 1800-1803.

3. Low TLK, Thurman GB, McAdoo M, et al. The chemistry and biology of thymosin. I. Isolation, characterization, and biological activities of thymosin $\alpha 1$ and polypeptide $\alpha 1$ from calf thymus. J Biol Chem. 1979; 254(3): 981-986.

4. Caldarella J, Goodall GJ, Felix AM, et al. Thymosin $\alpha 11$ : A peptide related to thymosin $\alpha 1$ isolated from calf thymosin fraction 5 . Proc Nat Acad Sci USA. 1983; 80(24): 7424-7427.

5. Low TLK, Goldstein AL. Thymic hormones: an overview. In G. D. Sabato, J. J. Langone, \& H. V. Vunakis (Eds.), Methods in enzymology. 1985; vol. 116 (pp 213-219). San Diego: Academic Press, Inc.

6. Freire $\mathrm{M}$, Crivellaro $\mathrm{O}$, Isaacs $\mathrm{C}$, et al. Translation of mRNA from calf thymus in the wheat germ system: Evidence for a precursor of thymosin $\alpha 1$. Proc Nat Acad Sci USA. 1978; 75(12): 6007-6011.

7. Freire M, Hannappel E, Rey M, et al. Purification of thymus mRNA coding for a 16,000 dalton polypeptide containing the thymosin $\alpha 1$ sequence. Proc Nat Acad Sci USA. 1981; 78(1): 192-195.

8. Haritos AA, Goodall GJ, Horecker BL. Prothymosin $\alpha$ isolation and properties of the major immunoreactive form of thymosin $\alpha 1$ in rat thymus. Proc Nat Acad Sci USA. 1984; 81(4): 1008-1011.

9. Goodall GJ, Dominguez F, Horecker BL. Molecular cloning of cDNA for human prothymosin $\alpha$. Proc Nat Acad Sci USA. 1986; 83(23): 89268928.

10. Haritos AA, Tsolas O, Horecker BL. Distribution of prothymosin $\alpha$ in rat tissues. Proc Nat Acad Sci USA. 1984; 81(5): 1391-1393.

11. Franco FJ, Díaz C, Barcia M, et al. Thymosin $\alpha 1$ is a native peptide in several tissues. Biochim Biophys Acta. 1992; 1120(1): 43-48.

12. Szabo, Panneerselvam, Clinton, et al. Prothymosin alpha gene in humans: organization of its promoter region and localization to chromosome 2. Hum Genet. 1993; 90(6): 629-34.

13. Gómez-Márquez J, Segade F, Dosil M, et al. The expression of prothymosin $\alpha$ gene in T lymphocytes and leukemic lymphoid cells is tied to lymphocyte proliferation. J Biol Chem. 1989; 264(15): 84518454.

14. Bustelo XR, Otero A, Gómez-Márquez J, et al. Expression of the rat prothymosin $\alpha$ gene during T-lymphocyte proliferation and liver regeneration. J Biol Chem. 1991; 266(3): 1443-1447.

15. Eschenfeldt WH, Manrow RE, Krug MS, et al. Isolation and partial sequencing of the human prothymosin $\alpha$ gene family. Evidence against export of the gene products. J Biol Chem. 1989; 264(13): 7546-7555.

16. Nogueira M, Freire M. Characteristics of the translation of thymosin alpha 1 precursor mRNA by cell-free wheat germ system. Evidence for the acetylation of thymosin alpha 1 precursor. Int J Biochem. 1985; 17(4): 533-536.

17. Conteas CN, Mutchnick MG, Palmer KC, et al. Cellular levels of thymosin immunoreactive peptides are linked to proliferative events: evidence for a nuclear site of action. Proc Nat Acad Sci. USA. 1990; 87(9): 3269-3273.

18. Moreira D, Díaz-Jullien C, Sarandeses CS, et al. The influence of phosphorylation of prothymosin $\alpha$ on its nuclear import and antiapoptotic activity. Biochem. Cell Biol. 2013; 91: 265-269.

19. Manrow RE, Sburlati AR, Hanover JA, et al. Nuclear targeting of prothymosin $\alpha$. J Biol Chem.1991; 266(6): 3916-3924.

20. Covelo G, Sarandeses CS, Díaz-Jullien C, et al. Prothymosin $\alpha$ interacts with free core histones in the nucleus of dividing cells. J Biochem. 2006; 140(5): 627-637.

21. Freire J, Covelo G, Sarandeses C, et al. Identification of nuclearimport and cell-cycle regulatory proteins that bind to prothymosin $\alpha$. Biochem Cell Biol. 2001; 79: 123-131.

22. Potaczek DP, Harb H, Michel S, et al. Epigenetics and allergy: from basic mechanisms to clinical applications. Epigenomics. 2017; 9(4): 539-571.

23. Sarandeses CS, Covelo G, Díaz-Jullien C, et al. Prothymosin $\alpha$ is processed to thymosin $\alpha 1$ and thymosin $\alpha 11$ by a lysosomal asparaginyl endopeptidase. I Biol Chem. 2003; 278(15): 1328613293.

24. Romani L, Oikonomou V, Moretti S, et al. Thymosin $\alpha 1$ represents a potential potent single-molecule-based therapy for cystic fibrosis. Nat Med. 2017; 23: 590-600.

25. Barcia MG, Castro JM, Jullien CD, et al. Prothymosin $\alpha$ is phosphorylated in proliferating stimulated cells. J Biol Chem. 1993; 268(7): 47044708.

26. Pérez-Estévez A, Díaz-Jullien C, Covelo G, et al. A 180-kDa protein kinase seems to be responsible for the phosphorylation of prothymosin $\alpha$ observed in proliferating cells. J Biol Chem. 1997; 272(16): 10506-10513.

27. Díaz-Jullien C, Moreira D, Sarandeses CS, et al. The M2-type isoenzyme of pyruvate kinase phosphorylates prothymosin alpha in proliferating lymphocytes. Biochem Biophys Acta. 2011; 1814: 355-365.

28. Freire M, Sarandeses CS, Covelo G, et al. Phosphorylation of Prothymosin $\alpha$. An Approach to Its Biological Significance. In Thymosins (Gerald Litwack, ed.). 2016; vol. 102: pp. 73-99. Academic Press, UK

29. Jiang X, Kim HE, Shu H, et al. Distinctive roles of PHAP proteins and prothymosin alpha in a death regulatory pathway. Science. 2003; 299: 223-226.

30. Fan Z, Beresford PJ, Oh DY, et al. Tumor suppressor NM23-H1 is a granzyme A-activated DNase during CTL-mediated apoptosis, and the nucleosome assembly protein SET is its inhibitor. Cell. 2003; 112: 659-672

31. Shen SM, Yu Y, Wu YL, et al. Downregulation of ANP32B, a novel substrate of caspase-3, enhances caspase-3 activation and apoptosis induction in myeloid leukemic cells. Carcinogenesis. 2010; 31: 419-426.

32. Matilla A, Radrizzani M. The Anp32 family of proteins containing leucine-rich repeats. Cerebellum. 2005; 4: 7-18.

33. Roth W, Wagenknecht B, Klumpp A, et al. APRIL, a new member of the tumor necrosis factor family, modulates death ligand-induced apoptosis. Cell Death Differ. 2001; 8: 403-410. 
34. Adachi Y, Pavlakis GN, Copeland TD. Identification of in vivo phosphorylation sites of SET, a nuclear phosphoprotein encoded by the translocation breakproint in acute undifferentiated leukemia FEBS Lett. 1994; 340: 231-235

35. Barbeito P, Sarandeses CS, Díaz-Jullien C, et al. Prothymosin $\alpha$ interacts with SET, ANP32A and ANP32B and other cytoplasmic and mitochondrial proteins in proliferating cells. Arch Biochem Biophys. 2017; 635: 74-86.
36. Karetsou Z, Martic G, Tavoulari S, et al. Prothymosin $\alpha$ associates with the oncoprotein SET and is involved in chromatin decondensation. FEBS Lett. 2004; 577: 496-500.

37. Seo SB, McNamara P, Heo S, et al. Regulation of histone acetylation and transcription by INHAT, a human cellular complex containing the set oncoprotein. Cell. 2001; 104: 119-130.

38. Tang HL, Le AHP, Lung HL. The increase in mitochondrial association with actin precedes Bax translocation in apoptosis. Biochem J. 2006; 396: 1-5. 